\title{
A importância da saúde bucal em idosos hospitalizados: revisão integrativa
}

\author{
The importance of oral health in hospitalized elderly: an integrative review
}

\section{La importancia de la salud oral en adultos mayores hospitalizados: revisión integrada}

Wilton Jerônimo Dias ${ }^{1}$, Adriano Silva Araújo ${ }^{1}$, Pedro Henrique da Hora Sales ${ }^{1}$, Patrícia Mares de Miranda $^{2,3}$, Angélica Patricia León Gaines ${ }^{4}$, Ellen Karla Nobre dos Santos-Lima ${ }^{1,3 *}$.

\section{RESUMO}

Objetivo: Revisar a literatura científica atual sobre a atuação do cirurgião-dentista (CD) em ambiente hospitalar, incluindo em Unidade de Terapia Intensiva (UTI), para prevenção e tratamento de doenças bucais e agravos sistêmicos em indivíduos idosos. Métodos: Esta revisão integrativa foi realizada utilizando artigos disponíveis na base de dados online da Biblioteca Virtual em Saúde (BVS). Os descritores "idoso", "saúde bucal" e "hospitalar" (MESH / DeCS) foram utilizados para a busca na BVS com a estratégia "idoso AND saúde bucal AND hospitalar". Os artigos analisados por três avaliadores foram incluídos no estudo quando relacionados à saúde bucal do idoso no ambiente hospitalar, buscando informações sobre UTI. Resultados: Os trabalhos analisados não especificaram informações sobre UTI, mas apontaram a necessidade da inserção do CD na rede de cuidado hospitalar e a importância de outros estudos epidemiológicos no âmbito da Odontologia Hospitalar. Considerações finais: A saúde bucal pode evitar agravos em pacientes hospitalizados e, por isso, a presença de um CD em ambiente hospitalar pode contribuir para uma melhor recuperação do quadro geral do paciente. Para tal, o CD precisa ser qualificado e inserido à equipe multiprofissional.

Palavras-chave: Saúde bucal, Odontogeriatria, Odontologia geriátrica, Odontologia hospitalar, Atenção integral ao idoso.

\section{ABSTRACT}

Objective: The present study aimed to review the scientific literature about Dentistry in the hospital environment, including intensive care, for the prevention and treatment of oral diseases in elderly. Methods: This integrative review was carried out on articles available in the Biblioteca Virtual em Saúde (BVS) online database, found through the combined uniterms (MESH) "elderly", "oral health" and "hospital", published from 2016 to 2021 in English and Portuguese. The articles were analyzed by three evaluators and they were included in the study when related to the oral health of the elderly in the hospital environment, searching information about the intensive care. Results: The analyzed studies did not specify information about the intensive care but they pointed out the need to insert the dentist in the hospital care network and the importance of other epidemiological studies in the field of Hospital Dentistry. Final considerations: Promoting oral health prevents injuries in hospitalized elderly patients and, therefore, the presence of a dentist can positivily contribute to the general health status of hospitalized elderly. For this, the dentist needs to be qualified and integrated into the multiprofessional team.

Keywords: Oral health, Geriatric dentistry, Hospital dentistry, Comprehensive health care.

\section{RESUMEN}

Objetivo: Revisar la literatura científica actual sobre el desempeño de los odontólogos, en ambiente intrahospitalario, incluso dentro de la Unidad de Cuidados Intensivos (UCI), para la prevención y tratamiento de enfermedades bucodentales y afecciones sistémicas en adultos mayores. Métodos: Esta revisión integradora se realizó utilizando artículos disponibles en la base de datos de la Biblioteca Virtual en Salud (BVS) en línea. Como descriptores se utilizaron las palabras "adulto mayor", "salud oral" y "hospitalización" (MESH / DeCS) para la búsqueda en la BVS. Los artículos fueron analizados y seleccionados por tres evaluadores, y solo fueron incluidos en éste estudio, cuando estaban relacionados con "salud oral de adultos mayores en el ámbito hospitalario, internados en UCl". Resultados: Los estudios analizados no especificaron información sobre la UCI, pero señalaron la necesidad de la inserción de odontólogos en la red asistencial hospitalaria y la importancia de realizar otros estudios epidemiológicos en el campo de la Odontología Hospitalaria. Consideraciones finales: La salud oral puede prevenir lesiones en pacientes hospitalizados y, por tanto, la presencia de un odontólogo, en un ambiente hospitalario puede contribuir a una mejor recuperación del estado general del paciente. Para ello, el odontólogo necesita estar calificado e incluido en el equipo multiprofesional.

Palabras clave: Salud oral, Odontogeriatría, Odontología geriátrica, Odontología intrahospitalaria, Atención integral al adulto mayor.

\footnotetext{
${ }^{1}$ Centro Universitário Mario Pontes Jucá (UMJ), Maceió - AL. *E-mail: ellen.nobre@umj.edu.br

2 Centro Universitário Regional do Brasil (UNIRB), Salvador - BA.

3 Universidade Federal da Bahia (UFBA), Salvador - BA.

${ }^{4}$ Fundación Universitaria San Martín (FUSM), Bogotá, Colômbia.
} 


\section{INTRODUÇÃO}

A projeção populacional do Instituto Brasileiro de Geografia e Estatística (IBGE) aponta para o acelerado processo de envelhecimento da população brasileira nas próximas décadas (BRASIL, 2020). Por isso, há a necessidade de proporcionar maior qualidade de vida ao segmento idoso da população. O estado de saúde bucal dos idosos tem adquirido maior importância nas últimas décadas, visto que essa faixa da população é crescente em razão do aumento da expectativa de vida (VASCONCELOS AKM, et al., 2018).

Com o envelhecimento populacional, o conceito de qualidade de vida torna-se mais importante. E a saúde bucal tem um papel relevante, podendo influenciar o nível nutricional, o bem-estar físico, mental e o prazer de uma vida social ativa. A terceira idade é formada por um grupo heterogêneo de pessoas em virtude das diferentes experiências de vida acumuladas pelo indivíduo. Existem idosos de diferentes níveis econômicos, culturais e de saúde, além de idosos com diferentes níveis de motivação quanto à manutenção da saúde bucal. Dessa forma, essas diferenças podem afetar a adesão ao tratamento (LIU YCG, et al., 2021).

Em paralelo a essa realidade, é importante que a Odontologia se integre ao atendimento dos pacientes hospitalizados, inclusive nas Unidades de Terapia Intensiva (UTI), minimizando o risco de disseminação de patógenos da cavidade bucal que possam causar agravos sistêmicos, atuando na manutenção da higienização da cavidade bucal e controle de colonização intensa de patógenos (AMARAL COF, et al., 2013). As infecções de origem nosocomial estão entre as principais causas de mortalidade dos pacientes graves e a boca de tais pacientes pode ser um reservatório para patógenos, como exemplo, aqueles associados à pneumonia nosocomial (JOSHI M, et al., 2019).

A Odontologia Hospitalar objetiva promoção da saúde; prevenção, diagnóstico e tratamento de doenças orofaciais, de manifestações bucais de doenças sistêmicas e de consequências do tratamento das alterações sistêmicas. Foi reconhecida pelo Conselho Federal de Odontologia (CFO) em 2015. Nessa área de atuação, o Cirurgião-Dentista (CD) habilitado acolhe pacientes que necessitam de atendimento em ambiente hospitalar, internados ou não, e pacientes em assistência domiciliar (BRASIL, 2015; BRASIL, 2019).

Segundo Ticianel AK, et al. (2020), desde 2008, o Projeto de Lei (PL) № 2776/2008 foi apresentado à câmara dos deputados para tornar obrigatória a presença de CD nas UTIs. Tal PL foi substituído pelo PL da Câmara (PLC) № 34/2013 (vetado em 2019) para tornar obrigatória a prestação de assistência odontológica a pacientes em regime de internação hospitalar, aos portadores de doenças crônicas e aos atendidos em regime domiciliar na modalidade home care. O projeto prevê a redução de $A$ ) tempo de internação, B) riscos de infecção e C) gastos hospitalares. Além disso, a atuação do CD permite o diagnóstico precoce de doenças graves e a melhoria na qualidade de vida do paciente. Portanto, apesar do veto por questão econômica, o CFO ressalta que a assistência odontológica em ambiente hospitalar permite baixo investimento se comparado com o retorno em saúde.

Desse modo, o presente trabalho objetivou revisar a literatura científica sobre a atuação do CD em ambiente hospitalar, incluindo em UTI, para prevenção e tratamento de doenças bucais e agravos sistêmicos em indivíduos idosos, além de observar a atuação multidisciplinar do CD em ambiente hospitalar.

\section{MÉTODOS}

A revisão integrativa da literatura científica foi realizada em artigos disponíveis na base de dados online Biblioteca Virtual em Saúde (BVS), a qual inclui as bases Bibliografia Brasileira de Odontologia (BBO), ScIELO, LILACS, MEDLINE, Cochrane. Os descritores "idoso", "saúde bucal" e "hospitalar" estão presentes no Descritores em Ciências da Saúde (DeCS) e foram utilizados para a busca na BVS na estratégia "idoso $A N D$ saúde bucal $A N D$ hospitalar". Os artigos foram selecionados por dois pesquisadores de modo independente e, posteriormente, um terceiro avaliador experiente, revisou as escolhas.

Os critérios de inclusão foram: texto completo, idiomas português e inglês, ano de publicação 2016 a 2021; a partir do reconhecimento do exercício da Odontologia Hospitalar pelo Conselho Federal de Odontologia (CFO). Os trabalhos analisados pelos três avaliadores foram incluídos no estudo quando relacionados à 
saúde bucal do idoso no ambiente hospitalar, buscando informações sobre a unidade de terapia intensiva. Os critérios de exclusão foram: trabalhos em outras temáticas e trabalhos que não abordaram, simultaneamente, a saúde bucal do idoso no ambiente hospitalar.

A busca na BVS (realizada até janeiro de 2021) resultou em 82 trabalhos, dos quais 29 foram selecionados e 13 foram incluídos por trazerem, simultaneamente, informações relativas à saúde bucal do indivíduo idoso no ambiente hospitalar. A Figura 1 traz o fluxo da presente revisão integrativa da literatura científica.

Figura 1 - Fluxo da revisão integrativa da literatura científica.
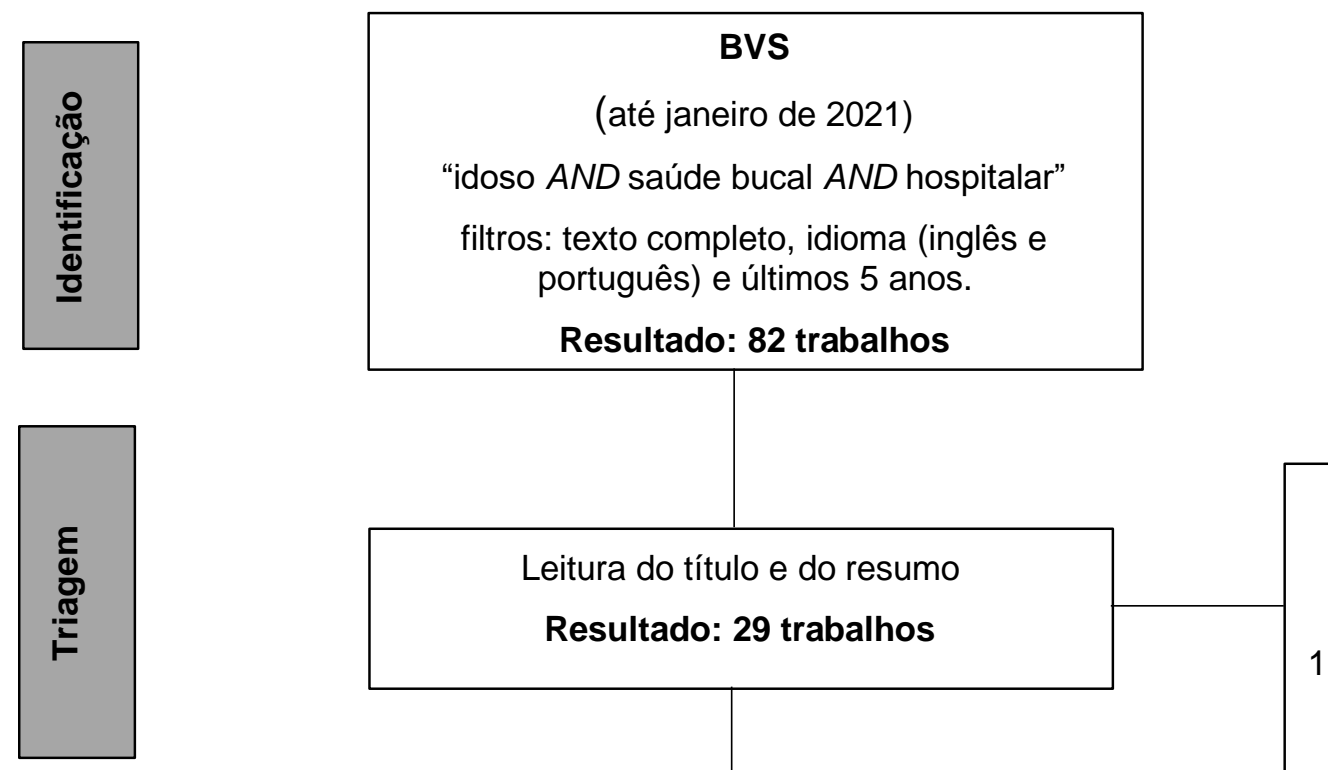

\section{Excluídos}

1. Trabalhos em outras temáticas
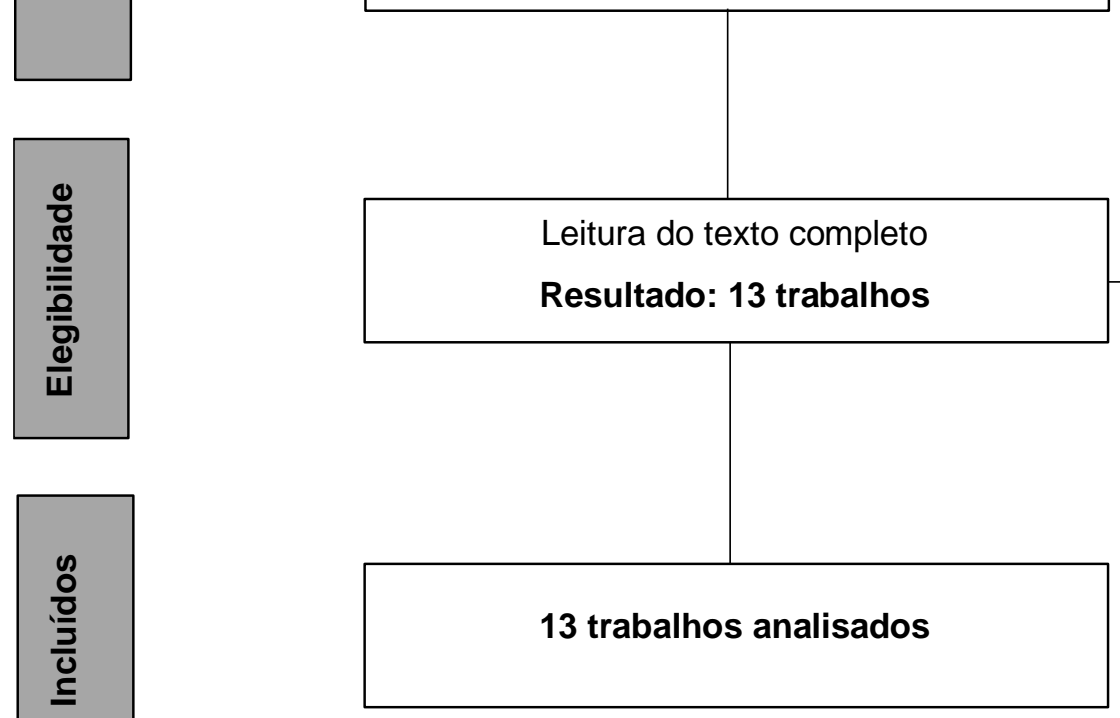

2. Trabalhos que não abordaram, simultaneamente, a saúde bucal do indivíduo idoso no ambiente hospitalar.

Fonte: Dias WJ, et al., 2021; baseado em Moher D, et al., 2009.

\section{RESULTADOS E DISCUSSÃO}

As principais informações das 13 publicações analisadas foram organizadas no Quadro 1, bem como sua aplicação no presente estudo. Os trabalhos analisados não especificaram informações sobre a Unidade de Terapia Intensiva, mas apontaram a necessidade da inserção do CD e a importância de outros estudos epidemiológicos no âmbito da Odontologia Hospitalar. 
Quadro 1 - Publicações incluídas no trabalho e aplicação no presente estudo.

\begin{tabular}{|c|c|c|c|c|c|c|}
\hline $\mathbf{N}$ & $\begin{array}{l}\text { Base de } \\
\text { Dados }\end{array}$ & Título & Autores (Ano) & Objetivo & Tipo de Estudo & Principal Achado / Aplicação \\
\hline 1 & LILACS & $\begin{array}{c}\text { Aspectos da assistência } \\
\text { odontológica a pacientes internados } \\
\text { em uma clínica médica de um } \\
\text { hospital universitário. }\end{array}$ & $\begin{array}{l}\text { MACEDO MP, et al. } \\
(2020)\end{array}$ & $\begin{array}{l}\text { Caracterizar o perfil sistêmico e } \\
\text { bucal de pacientes atendidos em } \\
\text { clínica médica de um Hospital } \\
\text { Universitário do Sistema de Saúde } \\
\text { Pública do Brasil. }\end{array}$ & $\begin{array}{l}\text { Estudo } \\
\text { observacional } \\
\text { retrospectivo }\end{array}$ & $\begin{array}{l}\text { Os resultados apontam para a necessidade de } \\
\text { atuação odontológica com atividades } \\
\text { preventivas no ambiente hospitalar. }\end{array}$ \\
\hline 2 & MEDLINE & $\begin{array}{c}\text { Má saúde bucal e mortalidade em } \\
\text { pacientes geriátricos internados em } \\
\text { um hospital de urgência: um estudo } \\
\text { observacional. }\end{array}$ & $\begin{array}{l}\text { MAEDA K e MORI N } \\
(2020)\end{array}$ & $\begin{array}{c}\text { Determinar a mortalidade intra- } \\
\text { hospitalar avaliando a saúde bucal } \\
\text { precária de pacientes geriátricos em um } \\
\text { hospital de cuidados agudos. }\end{array}$ & $\begin{array}{l}\text { Estudo } \\
\text { observacional } \\
\text { retrospectivo }\end{array}$ & $\begin{array}{l}\text { Em pacientes geriátricos, a saúde bucal } \\
\text { deficiente na admissão hospitalar foi um } \\
\text { preditor independente de mortalidade intra- } \\
\text { hospitalar em um hospital de cuidados agudos. }\end{array}$ \\
\hline 3 & MEDLINE & $\begin{array}{l}\text { Protocolo de atendimento clínico } \\
\text { para prevenção de mediastinite após } \\
\text { cirurgia de revascularização do } \\
\text { miocárdio: uma iniciativa de melhoria } \\
\text { de qualidade de um hospital privado. }\end{array}$ & $\begin{array}{l}\text { MACEDO TA, et al. } \\
(2019)\end{array}$ & $\begin{array}{l}\text { Descrever o efeito de um protocolo de } \\
\text { atendimento clínico padronizado para } \\
\text { prevenir a mediastinite em pacientes } \\
\text { submetidos à cirurgia de } \\
\text { revascularização do miocárdio (CRM). }\end{array}$ & $\begin{array}{l}\text { Estudo de } \\
\text { incidência }\end{array}$ & $\begin{array}{l}\text { A implementação do protocolo padronizado foi } \\
\text { associada a uma redução significativa na } \\
\text { incidência de mediastinite após CRM e } \\
\text { redução da mortalidade no grupo de pacientes } \\
\text { com mediastinite. }\end{array}$ \\
\hline 4 & MEDLINE & $\begin{array}{c}\text { O estado de saúde bucal } \\
\text { prejudicado na admissão está } \\
\text { associado a desfechos clínicos ruins } \\
\text { em pacientes pós-agudos } \\
\text { internados: um estudo de coorte } \\
\text { prospectivo. }\end{array}$ & $\begin{array}{l}\text { SHIRAISHI A, et al. } \\
\qquad(2019)\end{array}$ & $\begin{array}{l}\text { Investigar o impacto do estado de } \\
\text { saúde bucal prejudicado nos resultados } \\
\text { clínicos e funcionais na reabilitação } \\
\text { intra-hospitalar aguda. }\end{array}$ & $\begin{array}{l}\text { Estudo de coorte } \\
\text { prospectivo }\end{array}$ & $\begin{array}{c}\text { A deteç̧ão precoce de problemas de saúde } \\
\text { bucal e o tratamento por profissionais da área } \\
\text { odontológica, ou por meio da cooperação entre } \\
\text { profissionais médicos e odontológicos, devem } \\
\text { ser implementados em pacientes } \\
\text { hospitalizados em reabilitação pós reabilitação } \\
\text { aguda. }\end{array}$ \\
\hline 5 & $\begin{array}{l}\text { LILACS } \\
\text { BDENF }\end{array}$ & $\begin{array}{l}\text { O cuidado de enfermagem à saúde } \\
\text { bucal do idoso hospitalizado. }\end{array}$ & $\begin{array}{l}\text { FONSECA EOS } \\
\qquad(2019)\end{array}$ & $\begin{array}{c}\text { Analisar como é desenvolvido o cuidado } \\
\text { de enfermagem à saúde bucal em } \\
\text { idosos hospitalizados. }\end{array}$ & $\begin{array}{l}\text { Estudo descritivo, } \\
\text { exploratório e } \\
\text { qualitativo }\end{array}$ & $\begin{array}{l}\text { Foi possível revelar fragilidades na assistência } \\
\text { de enfermagem aos idosos hospitalizados. }\end{array}$ \\
\hline 6 & MEDLINE & $\begin{array}{l}\text { Efeito de uma intervenção mínima } \\
\text { massiva em pacientes idosos } \\
\text { hospitalizados com } \\
\text { disfagia orofaríngea: um estudo de } \\
\text { prova de conceito. }\end{array}$ & $\begin{array}{l}\text { MARTíN A, et al. } \\
\qquad(2018)\end{array}$ & $\begin{array}{c}\text { Avaliar o efeito de uma Intervenção } \\
\text { Mínima Massiva (MMI) na redução de } \\
\text { complicações nutricionais e } \\
\text { respiratórias em pacientes idosos } \\
\text { hospitalizados com disfagia orofaríngea } \\
\end{array}$ & $\begin{array}{l}\text { Ensaio clínico } \\
\text { controlado }\end{array}$ & $\begin{array}{l}\text { A MMI em pacientes idosos hospitalizados com } \\
\text { disfagia orofaríngea melhorou o estado } \\
\text { nutricional e a funcionalidade e reduziu } \\
\text { reinternações hospitalares, infecções } \\
\text { respiratórias e mortalidade. }\end{array}$ \\
\hline 7 & $\begin{array}{l}\text { LILACS } \\
\text { BDENF }\end{array}$ & $\begin{array}{l}\text { Construção e validação de um guia } \\
\text { educativo para cuidadores de } \\
\text { idosos em contexto hospitalar. }\end{array}$ & $\begin{array}{l}\text { XAVIER- } \\
\text { JÚNIOR JA, et al. } \\
\quad(2018)\end{array}$ & $\begin{array}{c}\text { Construir um guia educativo para o } \\
\text { cuidador de idosos atendidos em } \\
\text { ambiente hospitalar, com orientações } \\
\text { sobre: recursos materiais empregados } \\
\text { na rotina de higiene bucal; os cuidados } \\
\text { com as próteses dentárias e } \\
\text { como manter a saúde bucal. }\end{array}$ & $\begin{array}{l}\text { Estudo de } \\
\text { validação }\end{array}$ & $\begin{array}{c}\text { A elaboração do guia pode contribuir para a } \\
\text { execução do cuidado com a pessoa idosa, } \\
\text { minimizando dificuldades nas ações de higiene } \\
\text { bucal e nos cuidados com as próteses } \\
\text { dentárias, resultando em uma melhor } \\
\text { qualidade de vida. }\end{array}$ \\
\hline
\end{tabular}




\begin{tabular}{|c|c|c|c|c|c|c|}
\hline $\mathbf{N}$ & $\begin{array}{l}\text { Base de } \\
\text { Dados }\end{array}$ & Título & Autores (Ano) & Objetivo & Tipo de Estudo & Principal Achado / Aplicação \\
\hline 8 & MEDLINE & $\begin{array}{l}\text { Saúde bucal e dor orofacial em } \\
\text { pessoas com demência internadas } \\
\text { em enfermarias de hospitais } \\
\text { agudos: estudo de coorte } \\
\text { observacional. }\end{array}$ & $\begin{array}{l}\text { VAN DE RIJT LJM, } \\
\text { et al. (2018) }\end{array}$ & $\begin{array}{c}\text { Investigar a prevalência de dor orofacial } \\
\text { em pessoas com demência em } \\
\text { hospitais agudos no Reino Unido. }\end{array}$ & Estudo transversal & $\begin{array}{l}\text { Melhorar a higiene bucal em pacientes } \\
\text { hospitalares agudos com demência, } \\
\text { particularmente aqueles que não podem relatar } \\
\text { a dor, pode reduzir significativamente a dor e o } \\
\text { sofrimento nessa população. }\end{array}$ \\
\hline 9 & MEDLINE & $\begin{array}{l}\text { Efeito duradouro de um programa de } \\
\text { higiene bucal para pacientes com } \\
\text { acidente vascular cerebral durante a } \\
\text { reabilitação hospitalar: um ensaio } \\
\text { clínico randomizado de centro único. }\end{array}$ & KIM EK, et al. (2017) & $\begin{array}{c}\text { Implementar um programa de cuidados } \\
\text { de higiene bucal (PCHB) para pacientes } \\
\text { internados com AVC e avaliar sua } \\
\text { persistência após a alta. }\end{array}$ & $\begin{array}{l}\text { Ensaio clínico } \\
\text { randomizado }\end{array}$ & $\begin{array}{l}\text { Um PCHB conduzido durante a reabilitação } \\
\text { intra-hospitalar foi eficaz na melhoria da saúde } \\
\text { bucal e no desempenho do controle do biofilme } \\
\text { em pacientes com AVC, com efeitos ainda } \\
\text { observados três meses após a alta hospitalar. }\end{array}$ \\
\hline 10 & MEDLINE & $\begin{array}{l}\text { Higiene oral e cuidados com a boca } \\
\text { para idosos em hospitais agudos: } \\
\text { parte } 1\end{array}$ & STEEL BJ (2017a) & $\begin{array}{c}\text { Explorar a higiene oral e a provisão de } \\
\text { cuidados com a boca para idosos em } \\
\text { hospitais de urgência. }\end{array}$ & $\begin{array}{l}\text { Revisão da } \\
\text { literatura }\end{array}$ & $\begin{array}{c}\text { Os adultos mais velhos internados em } \\
\text { hospitais de urgência apresentaram alta } \\
\text { prevalência de doenças oro-dentais e } \\
\text { necessidades de cuidados bucais, mas a } \\
\text { prestação de cuidados mostrou-se abaixo do } \\
\text { ideal no Reino Unido. }\end{array}$ \\
\hline 11 & MEDLINE & $\begin{array}{l}\text { Higiene oral e cuidados com a boca } \\
\text { para idosos em hospitais agudos: } \\
\text { parte } 2\end{array}$ & STEEL BJ (2017b) & $\begin{array}{c}\text { Explorar a higiene oral e a provisão de } \\
\text { cuidados com a boca para idosos em } \\
\text { hospitais de urgência: recomendações } \\
\text { clínicas. }\end{array}$ & $\begin{array}{l}\text { Guia de prática } \\
\text { clínica }\end{array}$ & $\begin{array}{c}\text { Existe grande potencial para inovar e } \\
\text { desenvolver novas formas de cuidar de idosos } \\
\text { em hospitais de urgência. }\end{array}$ \\
\hline 12 & MEDLINE & $\begin{array}{l}\text { Eficácia de uma nova intervenção } \\
\text { pós-enxaguatório (“enxugar” mais } \\
\text { suplementos nutricionais orais) para } \\
\text { prevenção de pneumonia por } \\
\text { aspiração em pessoas idosas: um } \\
\text { ensaio randomizado multicêntrico } \\
\text { comparativo. }\end{array}$ & $\begin{array}{l}\text { HIGASHIGUCHI T, } \\
\text { et al. }(2017)\end{array}$ & $\begin{array}{l}\text { Avaliar a eficácia de uma nova } \\
\text { intervenção de higiene bucal: "enxugar" } \\
\text { mais suplementos nutricionais orais, } \\
\text { para prevenir a pneumonia por } \\
\text { aspiração em idosos. }\end{array}$ & $\begin{array}{l}\text { Ensaio clínico } \\
\text { controlado } \\
\text { multicêntrico }\end{array}$ & $\begin{array}{l}\text { O método de intervenção "limpar e fornecer } \\
\text { suplementos nutricionais orais" parece ajudar a } \\
\text { prevenir a pneumonia por aspiração, reduzindo } \\
\text { assim o risco de mortalidade. }\end{array}$ \\
\hline 13 & MEDLINE & $\begin{array}{l}\text { Fatores de risco para desnutrição } \\
\text { entre adultos mais velhos no } \\
\text { departamento de emergência: um } \\
\text { estudo multicêntrico. }\end{array}$ & $\begin{array}{l}\text { BURKS CE, et al. } \\
(2017)\end{array}$ & $\begin{array}{l}\text { Identificar fatores de risco modificáveis } \\
\text { associados à desnutrição em pacientes } \\
\text { idosos. }\end{array}$ & $\begin{array}{l}\text { Estudo transversal } \\
\text { multicêntrico }\end{array}$ & $\begin{array}{l}\text { Os fatores de risco que mais afetaram a dieta } \\
\text { foram problemas de saúde bucal, insegurança } \\
\text { alimentar e falta de transporte. }\end{array}$ \\
\hline
\end{tabular}

Fonte: Dias WJ, et al., 2021. 
Em estudo atual, com ferramenta validada, para determinar a mortalidade intra-hospitalar avaliando a saúde bucal precária de pacientes geriátricos em um hospital de cuidados agudos, observou-se que, em pacientes geriátricos, a saúde bucal deficiente na admissão hospitalar foi um preditor independente de mortalidade intra-hospitalar. Entretanto, os autores afirmam que estudos de intervenção com higiene bucal, estratificados por condições de saúde bucal, são necessários (MAEDA K e MORI N, 2020).

De acordo com Steel BJ (2017a), os adultos mais velhos internados em hospitais de urgência apresentaram alta prevalência de doenças oro-dentais e necessidades de cuidados bucais, mas a prestação de cuidados mostrou-se abaixo do ideal no Reino Unido. Segundo o autor, a literatura está crescendo, mas esta área ainda é relativamente negligenciada. Portanto, existe um grande potencial para desenvolver cuidados bucais em idosos em hospitais de urgência. Para tanto, Steel BJ (2017b) afirma que é necessária uma mudança na filosofia em saúde para incorporar os cuidados bucais como um componente essencial da prática holística. Dessa forma, mais pesquisas são necessárias para determinar as melhores maneiras de avaliar e tratar problemas bucais em pessoas idosas e promover e restaurar sua saúde bucal em hospitais.

No estudo realizado por Macedo MP, et al. (2020) para caracterizar o perfil sistêmico e bucal de pacientes atendidos em clínica médica de um Hospital Universitário do Sistema de Saúde Pública do Brasil, houve associação de diabetes e hipertensão na faixa etária de 60 a 75 anos de idade. Para a maioria dos pacientes estudados, a higiene bucal foi realizada menos que três vezes ao dia, sem uso de fio dental. Biofilme, cálculo dental, uso de próteses com higiene deficiente e estomatite protética foram registrados. Dentre os tratamentos realizados, destacaram-se terapia básica periodontal e exodontia.

Fonseca EOS (2019) buscou analisar como é desenvolvido o cuidado de enfermagem à saúde bucal em idosos hospitalizados e destacou que foi possível revelar fragilidades na assistência de enfermagem aos idosos hospitalizados. No entanto, tal estudo promoveu reflexões na assistência de enfermagem sobre os cuidados bucais do idoso hospitalizado.

Ao se investigar o impacto do estado de saúde bucal prejudicado nos resultados clínicos e funcionais na reabilitação intra-hospitalar aguda, observou-se que a detecção precoce de problemas de saúde bucal e o tratamento por profissionais da área odontológica, ou por meio da cooperação entre profissionais médicos e odontológicos, devem ser implementados em pacientes hospitalizados em reabilitação pós reabilitação aguda (SHIRAISHI A, et al., 2019). Um programa de cuidados de higiene bucal para pacientes internados com AVC conduzido durante a reabilitação intra-hospitalar foi eficaz na melhoria da saúde bucal e no desempenho do controle do biofilme em pacientes com AVC, com efeitos ainda observados três meses após alta hospitalar (KIM EK, et al., 2017).

Burks CE, et al. (2017) identificaram fatores de risco modificáveis associados à desnutrição em pacientes idosos. As características do paciente associadas à desnutrição incluíram não ter curso superior, ser internado em hospital e residir em casa de repouso. Dos fatores de risco examinados, aqueles que mais afetaram a dieta foram problemas de saúde bucal, insegurança alimentar e falta de transporte.

No contexto nutricional, Higashiguchi T, et al. (2017) avaliaram a eficácia de uma nova intervenção de higiene bucal associada a suplementos nutricionais orais para prevenir a pneumonia por aspiração em idosos. A intervenção adicional de cuidado bucal foi a higiene bucal com lenços umedecidos comerciais para enxugar a boca, todos os dias durante o período de intervenção, além da higiene bucal convencional fornecida pelos cuidadores de cada unidade. Tal método de intervenção ajudou a prevenir a pneumonia por aspiração, reduzindo assim o risco de mortalidade.

Ao se avaliar o efeito de uma Intervenção Mínima Massiva (MMI) na redução de complicações nutricionais e respiratórias em pacientes idosos hospitalizados com disfagia orofaríngea. Tal MMl em pacientes idosos hospitalizados com disfagia orofaríngea melhorou o estado nutricional e a funcionalidade e reduziu reinternações hospitalares, infecções respiratórias e mortalidade. A referida MMl constituiu-se em: A) espessamento de fluidos e alimentos com textura modificada; B) suplementação calórica e proteica; C) recomendações de saúde e higiene bucal durante a internação e após a alta (MARTíN A, et al., 2018).

$\mathrm{Na}$ investigação da prevalência de dor orofacial em pessoas com demência em hospitais agudos no Reino Unido, foi observada saúde bucal deficiente nos participantes dentados e edentados. Constatou-se preditores 
significativos para a presença de dor orofacial: frequência de escovação, indicação da qualidade da mastigação, consistência da comida, presença de anormalidades extraorais, pessoa que realizava o cuidado bucal e higiene bucal em participantes dentados. Nesse estudo, os participantes que não eram mais capazes de relatar a dor foram significativamente mais propensos a sentir dor orofacial. Portanto, melhorar a higiene bucal em pacientes hospitalares agudos com demência, particularmente aqueles que não podem relatar a dor, pode reduzir significativamente a dor e o sofrimento nessa população (VAN DE RIJT LJM, et al., 2018).

Macedo TA, et al. (2019) descreveram o efeito de um protocolo de atendimento clínico padronizado para prevenir a mediastinite em pacientes submetidos à cirurgia de revascularização do miocárdio. $O$ protocolo consistiu na utilização pelo paciente de kit contendo clorexidina $2 \%$ para banho, mupirocina $20 \mathrm{mg} / \mathrm{g}$ para uso tópico nasal e clorexidina $0,12 \%$ para higiene bucal por 5 dias antes da cirurgia, além de profilaxia com antimicrobiano glicopeptídeo e controle estrito da glicose (110-140 mg / dL) durante a cirurgia e pós-operatório imediato. A implementação do protocolo padronizado foi associada a uma redução significativa na incidência de mediastinite após cirurgia de revascularização do miocárdio e redução da mortalidade no grupo de pacientes com mediastinite.

Além das intervenções supracitadas, Steel BJ (2017b) elaborou um guia de prática clínica com recomendações clínicas para o profissional da saúde, o qual explorou a higiene oral e a provisão de cuidados com a boca aplicados aos idosos em hospitais de urgência. Ao passo que Xavier-Júnior JA, et al. (2018) propuseram construir um guia educativo para o cuidador de idosos atendidos em ambiente hospitalar, com orientações sobre A) recursos materiais empregados na rotina de higiene bucal; B) cuidados com as próteses dentárias e C) como manter a saúde bucal. Para os autores, o guia pode contribuir para a execução do cuidado com a pessoa idosa, resultando em uma melhor qualidade de vida, por minimizar dificuldades nas ações de higiene bucal e nos cuidados com as próteses dentárias.

Os trabalhos analisados não especificaram informações sobre a UTI. A carência de informações relativas à saúde bucal na UTI pode ser um reflexo da necessidade de maior inserção da Odontologia nesse ambiente e da necessidade de cursos que capacitem o CD; as quais podem ser consequência, no Brasil, da ausência de obrigatoriedade para a prestação de assistência odontológica a pacientes em regime de internação hospitalar (SIMÕES TC, et al., 2019; TICIANEL AK, et al., 2020).

Segundo Goronzy JJ e Weyand CM (2019), os idosos, naturalmente, apresentam uma resposta imune alterada devido à imunosenescência. Tal processo fisiológico aumenta o risco de infecções sistêmicas, cujos microrganismos causadores podem colonizar a cavidade oral. Algumas bactérias presentes na cavidade bucal, em condições normais, não são patogênicas, mas, com a debilitação imune do hospedeiro, podem favorecer a colonização de microrganismos patogênicos ou tornarem-se patogênicas, sendo documentada na literatura a relação de microrganismos bucais com endocardite bacteriana e pneumonia nosocomial (GAETTI-JARDI EC, et al., 2013; MOTHIBE JV e PATEL M, 2017; BURKS CE, et al., 2017; MACEDO MP, et al., 2020).

Dentes cariados, cálculo dentário, alimentação comprometida por falta de dentes, língua mal higienizada, doença gengival e mucosa bucal seca e avermelhada são comumente observados em idosos hospitalizados. Tais pacientes com a saúde fragilizada precisam realizar a higiene bucal para reduzir bacteremia, a qual, com frequência, precisa ser realizada por membro da família ou cuidador (BUENO AC, et al., 2012; GAETTI-JARDI EC, et al., 2013; HIGASHIGUCHI T, et al., 2017; XAVIER-JÚNIOR JA, et al., 2018; MACEDO MP, et al., 2020).

A Odontologia Hospitalar objetiva promover melhores condições de saúde e qualidade de vida, independente do seu nível de complexidade, estando o indivíduo sob o olhar de uma equipe multidisciplinar. A atuação odontológica está inserida em um contexto multiprofissional e o CD deve trabalhar com médicos, enfermeiros, psicólogos, fisioterapeutas e assistentes sociais. As intervenções odontológicas devem ocorrer desde a hospitalização, concomitantemente ao tratamento e após a alta hospitalar (GAETTI-JARDI EC, et al., 2013; MARTíN A, et al., 2018; FONSECA EOS, 2019; MACEDO TA, et al., 2019).

É importante destacar que, na cavidade bucal, existem áreas que facilitam a adesão de microrganismos, favorecendo a formação do biofilme. Sem a higiene bucal adequada, a quantidade e a patogenicidade do 
biofilme podem aumentar com o tempo de internação, podendo contribuir para a instalação ou agravamento das doenças bucais, disseminação de patógenos e estabelecimento de infecções nosocomiais (GAETTIJARDI EC, et al., 2013; KIM EK, et al., 2017; VAN DE RIJT LJM, et al., 2018; MACEDO TA, et al., 2019; MACEDO MP, et al., 2020).

O paciente que apresenta comprometimento sistêmico pode ter uma higienização bucal inadequada, havendo maior acúmulo de biofilme em restos radiculares e necrose pulpar, induzindo dispersão de patógenos por via orofaríngea e hematogênica. $A$ atuação hospitalar do $C D$ através de prevenção e tratamento evita agravo aos demais órgãos e sistemas, independente da complexidade do serviço, incluindo assistência paliativa hospitalar, na qual deve-se conservar, o quanto for possível, a aptidão de se nutrir via oral. Para pacientes que apresentam disfagia, sugere-se que uma dentição saudável seja conservada não apenas pela relevância para a saúde, mas para preservar a dignidade e a qualidade de vida (JALES SMCP, 2011; GAETTIJARDI EC, et al., 2013; KIM EK, et al., 2017; MARTíN A, et al., 2018; VAN DE RIJT LJM, et al., 2018; MACEDO TA, et al., 2019; MACEDO MP, et al., 2020).

Visto que os trabalhos analisados não especificaram a UTI, é importante ressaltar que os procedimentos de rotina nessa unidade hospitalar ajudam os microrganismos da cavidade bucal a se disseminar para outras partes do organismo. A ventilação mecânica, pode transportar bactérias presentes na cavidade bucal e da orofaringe até os pulmões, provocando a pneumonia nosocomial, aumentando o tempo de hospitalização e podendo levar o paciente a óbito. Dessa forma, a avaliação das condições bucais em pacientes internados na UTI pode contribuir para o controle de infecções no ambiente hospitalar. Esses pacientes necessitam de cuidados rigorosos devido a um quadro clínico caracterizado por imunodeficiência, sendo susceptíveis à instalação de infecções bucais e/ou sistêmicas, agravando o seu estado de saúde geral (AMARAL COF, et al., 2013).

O paciente pode ser dependente para realizar sua higienização bucal de forma adequada e eficiente, necessitando, assim, da presença do profissional devidamente capacitado ou bem orientado para adequação ou reestabelecimento da qualidade do meio bucal. Diante disso, justifica-se a presença de um CD qualificado como parte da equipe multidisciplinar (FONSECA EOS, 2019; SHIRAISHI A, et al., 2019).

As pneumonias nosocomiais são as infecções com maior relevância em pacientes hospitalizados. Logo, os pacientes que se encontram internados em UTI são mais suscetíveis à pneumonia nosocomial, sobretudo os pacientes que se encontram sob ventilação mecânica, com uma taxa de 50 a $80 \%$ de morbidade. (BUENO AC, et al., 2012).

Em sujeitos que se encontram em situação crítica, a avaliação cotidiana da orofaringe, por exemplo, e a conservação de uma higiene bucal aceitável são exercícios difíceis de se realizar, sobretudo quando o sujeito encontra em intubação orotraqueal, restringindo a atenção executada no leito. A equipe de saúde, portanto, pode ser guiada e motivada pelo $C D$, inclusive com a aplicação de métodos alternativos de higienização (JALES SMCP, 2011; HIGASHIGUCHI T, et al., 2017; FONSECA EOS, 2019).

A realização de intervenções educativas em saúde bucal pode reduzir as taxas de pneumonia associada à ventilação mecânica em pacientes na UTI. A interação entre $C D$, médico, fisioterapeuta, fonoaudiólogo e enfermeiro é importante nesse processo. Cada instituição deve elaborar e instituir um protocolo de higiene bucal de acordo com o perfil dos pacientes assistidos em UTI, em consonância com a Comissão de Controle de Infecção Hospitalar e o corpo de enfermagem (AMARAL COF, et al., 2013; STEEL BJ, 2017b; XAVIERJÚNIOR JA, et al., 2018).

É notório que diversos pacientes internados mostram situações orais pré-existentes e que necessitam ser previamente tratadas tanto pelos profissionais de saúde quanto pela família do paciente. Problemas orais como a doença periodontal são foco de microrganismos com ação sistêmica, pois induzem a produção de citocinas inflamatórias (BUENO AC, et al., 2012; HAJISHENGALLIS G, 2015; STEEL BJ, 2017a). Para pacientes que irão se submeter ao tratamento radioterápico, por exemplo, uma avaliação cotidiana da cavidade oral é necessária, com procedimentos profiláticos, curativos e restauradores; promovendo uma simbiose na microbiota bucal que favorece a saúde sistêmica (SCHERMA AP, 2013; HAJISHENGALLIS G, 2015). 


\section{CONSIDERAÇÕES FINAIS}

A partir desta revisão, observa-se que mais pesquisas são necessárias para promover e reabilitar a saúde bucal das pessoas idosas no ambiente hospitalar. Porém, atuar fora do consultório dentário continua sendo um grande desafio para o profissional da Odontologia, pois muitas instituições hospitalares não se encontram aptas a dar suporte a esse atendimento especializado. No entanto, a saúde bucal pode evitar agravos em pacientes hospitalizados e, por isso, a presença de um CD pode contribuir para uma melhor recuperação do quadro geral do paciente. Para tal, o CD precisa ser qualificado e inserido à equipe multiprofissional.

\section{AGRADECIMENTOS}

Os autores agradecem ao Centro Universitário Mario Pontes Jucá (UMJ, Maceió - AL) e à coordenação do curso de Odontologia (UMJ, Maceió - AL).

\section{REFERÊNCIAS}

1. AMARAL COF, et al. Importância do cirurgião dentista em Unidade de Terapia Intensiva: avaliação multidisciplinar. Revista de associação paulista de cirurgiões dentistas, 2013; 70(3): 207-211.

2. BRASIL. Conselho Federal de Odontologia. Resolução CFO-162/2015. Reconhece o exercício da Odontologia Hospitalar pelo cirurgião-dentista. Disponível em: https://website.cfo.org.br/wpcontent/uploads/2015/12/ResolucaoCFO-162-15.pdf. Acessado em: 17 de março de 2021.

3. BRASIL. Conselho Federal de Odontologia. Resolução CFO-163/2015. Conceitua a Odontologia Hospitalar e define a atuação do cirurgião-dentista habilitado a exercê-la. Disponível em: http://sistemas.cfo.org.br/visualizar/atos/RESOLU\%C3\%87\%C3\%83O/SEC/2015/163. Acessado em: 17 de março de 2021.

4. BRASIL. Conselho Federal de Odontologia. Resolução CFO-203/2019. Altera a Resolução CFO-162/2015 e dá outras providências. Disponível em: http://sistemas.cfo.org.br/visualizar/atos/RESOLU\%C3\%87\%C3\%83O/SEC/2019/203. Acessado em: 17 de março de 2021.

5. BRASIL. Conselho Federal de Odontologia. Resolução CFO-204/2019. Altera as alíneas do art. 2ํ da Resolução CFO163/2015. Disponível em: http://sistemas.cfo.org.br/visualizar/atos/RESOLU\%C3\%87\%C3\%83O/SEC/2019/204. Acessado em: 17 de março de 2021.

6. BRASIL. Instituto Brasileiro de Geografia e Estatística. Centro de Documentação e Disseminação de Informações. Brasil em números. v. 28. Rio de Janeiro: IBGE, 2020.

7. BUENO AC, et al. Associações entre Fatores de Risco e Complicações Bucais em Pacientes com Câncer de Cabeça e Pescoço Tratados com Radioterapia Associada ou não à Quimioterapia. Pesq Bras Odontoped Clin Integr., 2012; 14(3): 97-103.

8. BURKS CE, et al. Risk Factors for Malnutrition among Older Adults in the Emergency Department: A Multicenter Study. J Am Geriatr Soc., 2017; 65(8): 17411747.

9. FONSECA EOS. O cuidado de enfermagem à saúde bucal do idoso hospitalizado. 2019. Dissertação (Mestrado em Enfermagem e saúde) - Universidade Federal da Bahia. Salvador, 2019; 85f. Disponível em: https://repositorio.ufba.br/ri/handle/ri/30975. Acessado em: 05 de fevereiro de 2021.

10. GAETTI-JARDI EC, et al. Atenção odontológica a pacientes hospitalizados: revisão de literatura e proposta de protocolo de higiene oral. Rev. bras. Ciênc. Saúde, 2013; 11(35): 31-36.

11. GORONZY JJ, WEYAND CM. Mechanisms underlying T cell ageing. Nat Rev Immunol., 2019; 19(9):573-583.

12. HAJISHENGALLIS G. Periodontitis: from microbial immune subversion to systemic inflammation. Nat. Rev. Immunol., $2015 ; 15(1): 30-44$.

13. HIGASHIGUCHI T, et al. Efficacy of a New Post-Mouthwash Intervention (Wiping Plus Oral Nutritional Supplements) for Preventing Aspiration Pneumonia in Elderly People: A Multicenter, Randomized, Comparative Trial. Ann Nutr Metab., 2017; 71(3-4): 253-260.

14. JALES SMCP. Avaliação da efetividade de um protocolo de cuidados odontológicos no alívio da dor, sintomas bucais e melhora da qualidade de vida em pacientes com câncer de cabeça e pescoço em cuidados paliativos: Ensaio clínico não controlado. Tese - Faculdade de Medicina, Universidade de São Paulo. São Paulo, 2011. Disponível em: https://www.teses.usp.br/teses/disponiveis/5/5138/tde-08122011-

161547/publico/SumatraMeloCostaPereiraJales.pdf. Acessado em: 30 de outubro de 2020.

15. JOSHI M, et al. Nosocomial infection: source and prevention. Int J Pharm Sci \& Res., 2019; 10(4): $1613-24$.

16. KIM EK, et al. Lasting effect of an oral hygiene care program for patients with stroke during in-hospital rehabilitation: a randomized single-center clinical trial. Disabil. Rehabil., 2017; 39(22): 2324-2329. 
17. LIU YCG, et al. Update and review of the gerodontology prospective for 2020's: Linking the interactions of oral (hypo)functions to health vs. systemic diseases. J Dent Sci., 2021; 16(2): 757-773.

18. MACEDO MP, et al. Aspects of dental care for patients hospitalized in a medical clinic from a university hospital. ABCS Health Sci., 2020; 45: 1198.

19. MACEDO TA, et al. Clinical-care protocol for preventing mediastinitis after coronary artery bypass graft surgery: A quality improvement initiative from a private hospital. J. Card. Surg., 2019; 34(5): 274-278.

20. MAEDA K, MORI N._Poor oral health and mortality in geriatric patients admitted to an acute hospital: an observational study. BMC Geriatr., 2020; 20(26).

21. MARTÍN A, et al. Effect of A Minimal-Massive Intervention in Hospitalized Older Patients with Oropharyngeal Dysphagia: A Proof of Concept Study. J Nutr Health Aging., 2018; 22(6): 739-747.

22. MOHER D, et al. Preferred Reporting Items for Systematic Reviews and Meta-Analyses: The PRISMA Statement. PLoS Med., 2009; 6(7): e1000097.

23. MOTHIBE JV, PATEL M. Pathogenic characteristics of Candida albicans isolated from oral cavities of denture wearers and cancer patients wearing oral prostheses. Microb Pathog, 2017; 110: 128-134.

24. SCHERMA AP. Avaliação dos cuidados odontológicos pré e trans-tratamento radioterápico. Braz J Periodontol., 2013; 23(3): 30-38.

25. SHIRAISHI A, et al. Impaired oral health status on admission is associated with poor clinical outcomes in post-acute inpatients: A prospective cohort study. Clinical Nutrition, 2019; 38(6): 2677-2683.

26. SIMÕES TC, et al. Panorama situacional dos cursos de habilitação em odontologia hospitalar: revisão integrativa. Revista da ABENO, 2019; 19(4): 70-77.

27. STEEL BJ. Oral hygiene and mouth care for older people in acute hospitals: part 1. Nurs. Older People, 2017; 29(9): 26-31. a

28. STEEL BJ. Oral hygiene and mouth care for older people in acute hospitals: part 2. Nurs. Older People, 2017; 29(10): 20-25. b

29. TICIANEL AK, et al. Conselho Regional de Odontologia de Mato Grosso (CRO-MT). Manual de Odontologia Hospitalar. 2020. Disponível em: https://website.cfo.org.br/wp-content/uploads/2020/07/manual-odontologia-hospitalar.pdf. Acessado em: 17 de março de 2021.

30. VAN DE RIJT LJM, et al. Oral health and orofacial pain in people with dementia admitted to acute hospital wards: observational cohort study. BMC Geriatr., 2018; 18(1): 121.

31. VASCONCELOS AKM, et al. A ascensão da odontogeriatria no Brasil através do panorama de suas publicações / The rise of odontogeriatrics in Brazil through the panorama of its publications. Rev. pesqui. cuid. fundam. (Online), 2018; 10(3, n. esp): 165-171.

32. XAVIER-JÚNIOR JA, et al. Construção e validação de um guia educativo para cuidadores de idosos em contexto hospitalar. Rev. pesqui. cuid. fundam., 2018; 10(3): 317-321. 